\title{
La retención del personal para disminuir la deserción de la población millennial en el Ejército Nacional de Colombia ${ }^{1}$
}

https://doi.org/10.21830/9789585241459.05

\author{
Edna Jackeline Latorre Rojas ${ }^{2}$ \\ Escuela Militar de Cadetes "General José María Córdova” \\ Maira Gimena Botina Benavides ${ }^{3}$ \\ Jason Armando Reyes Arias ${ }^{4}$ \\ Martha Liliana Mendoza Sierra 5 \\ Dirección de Investigación, Innovación y Desarrollo \\ de Personal del Ejército Nacional
}

\section{Resumen}

Antecedentes: actualmente, más del $50 \%$ de los efectivos del Ejército Nacional pertenecen a la generación millennial y de acuerdo con cifras de la Bases de Datos de Personal del Ejército Nacional, al año 2016 se habían presentado en los últimos diez años un acumulado

1 Capítulo de libro que parte de los resultados del proyecto de investigación Hebe: Caracterización de los Factores Asociados a la Retención del Personal para Disminuir la Deserción de la Población Millennial en el Ejército Nacional de Colombia, del Grupo de Investigación en Ciencias Militares de la Escuela Militar de Cadetes “General José María Córdova” (ESMIC), registrado con el código COL0082556 de Minciencias. Los puntos de vista y los resultados de este capítulo pertenecen a los autores y no reflejan necesariamente los de las instituciones participantes.

2 Magíster en Educación e Investigación Universitaria de la Universidad Sergio Arboleda y especialista en Educación e Investigación Universitaria de la misma institución. Psicóloga de la Universidad Católica de Colombia. Investigadora del Grupo de Investigación en Ciencias Militares de la Escuela Militar de Cadetes “General José María Córdova” (ESMIC). Orcid: https://orcid.org/0000-0002-0301-9036. Contacto: edna.latorre@ esmic.edu.co

3 Subteniente del Ejército Nacional. Especialista en Gerencia de Proyectos de la Corporación Universitaria Minuto de Dios. Profesional en Administración de Empresas de la Universidad de Nariño. Diplomado en Identificación, Preparación y Evaluación de Proyectos de Inversión Pública en Metodología General Ajustada (MGA) y diplomado en Formulación de Proyectos de Innovación con Enfoque Social.

4 Especialista en Gerencia de la Calidad de la Universidad Militar Nueva Granada. Profesional en Administración Pública de la Escuela Superior de Administración Pública. Auditor certificado en norma ISO 9001:2015, ISO 14001:2015 e ISO 45001:2018 con énfasis en Procesos de Responsabilidad Social Empresarial de acuerdo con la norma ISO 26001 y Gestión Humana. Asesor de Evaluación y Seguimiento para el Departamento de Personal del Ejército Nacional.

5 Magíster en Dirección y Gestión de Proyectos de la Universidad Camilo José Cela, España. Profesional en Administración de Empresas de la Universidad Santo Tomas. Auditor interno en Sistema Integrado de Gestión certificado por Icontec. Asesora de la Dirección de Investigación, Innovación y Desarrollo de Personal del Ejército Nacional. 
estadístico de 58.000 procesos de retiro voluntario. Esta situación plantea la necesidad de retener a los mejores talentos a través de estrategias que generen atractivos capaces de satisfacer las demandas más tradicionales y los intereses más dinámicos. Objetivo: este trabajo caracteriza los factores asociados a la retención del personal de 209 militares oficiales millennial del Ejército Nacional y hace una propuesta de intervención para el mejoramiento de retención de la institución. Materiales y métodos: el marco metodológico fue mixto, de orden histórico hermenéutico. Inicialmente se realizó un análisis de datos con los principios de la teoría fundamentada y posteriormente se desarrolló una matriz de análisis sobre las categorías proyecto de vida y formación del contrato psicológico. Resultados: la institución debe tener en cuenta que la tipología militar oficial millennial se caracteriza por fundamentar su proyecto de vida alrededor del ámbito familiar, valora la estabilidad económica que le da la organización y exige un equilibrio entre el cumplimiento de la misión y su esfera personal. Conclusiones: se concluye que para disminuir la deserción de la población militar oficial millennial es necesario tener en cuenta factores asociados a la formación del contrato psicológico y articular los planes de retención de personal y demás procesos de gestión humana.

Palabras clave: contrato psicológico; deserción universitaria; oficiales militares; población millennial; proyecto de vida; retención de personal.

\section{Introducción}

Se considera que la administración del talento humano representa para las instituciones un factor decisivo entre el éxito o el fracaso, por tanto, resulta imprescindible observar, así como también estudiar las implicaciones que tienen las características de su población o sus colaboradores, particularmente centrar el interés en aquellos factores que como sociedad o generación traen consigo las personas.

Particularmente, cuando se habla de una institución militar como el Ejército Nacional, en la cual existe un fuerte tradicionalismo, caracterizado por el arraigo por creencias, hábitos y costumbres, en donde la disciplina, la obediencia, así como la subordinación son ejes fundamentales, queda abierta la discusión respecto de cómo lograr la incorporación y la adaptación de nuevas generaciones, que traen consigo amplios desafíos desde diferentes cosmovisiones y para las cuales puede que no sea atractivo mantenerse en un mismo puesto durante veinticinco años o más.

Actualmente, más del $50 \%$ de los efectivos del Ejército Nacional pertenecen a la generación millennial, que con sus características particulares conviven con las expectativas de retiro de los baby boomers y con el pensamiento progresivo de la generación X. La coexistencia de personas con marcadas diferencias, expectativas y formas de trabajar es sin duda el principal reto en el ciclo de la gestión del talento humano, que va desde la atracción, incorporación, retención y permanente motivación del personal hasta el retiro. 
En consecuencia, se ha planteado la necesidad de caracterizar a la población millennial de oficiales del Ejército Nacional, quienes tienen la responsabilidad de conducir y liderar a hombres y mujeres para desarrollar las diferentes misiones de defensa, seguridad y apoyo a la seguridad, con el objetivo de identificar factores motivacionales extrínsecos e intrínsecos que minimicen la probabilidad de retiros prematuros en la institución.

La importancia de este análisis radica en la problemática actual de retiro voluntario: de acuerdo con cifras de las Bases de Datos de Personal del Ejército Nacional, al año 2016 se habían presentado en los últimos diez años un acumulado estadístico de 58.000 procesos de retiro voluntario. Esta situación plantea la necesidad de retener a los mejores talentos a través de estrategias que generen mejores atractivos capaces de satisfacer las demandas más tradicionales y los intereses más dinámicos.

La tarea consiste, también, en optimizar la interrelación entre las diferentes generaciones presentes en la institución, de modo que aquellos con más experiencia compartan el conocimiento con los más jóvenes, así como estos también enseñen a sacar el mejor provecho de la tecnología y la innovación. Para esto se deben definir claramente los factores motivacionales y las expectativas que tienen los oficiales millennial sobre el Ejército Nacional desde su proyecto de vida a partir del contrato psicológico construido.

A partir de esto se debe comprender que la generación millennial está conformada por quienes nacieron con la consolidación de la era digital y su primera cohorte se hizo adulta en el cambio del milenio, es decir, se encuentra ubicada actualmente en un rango de edad de 21 a los 34 ańos y se consideran el $26 \%$ de la población mundial. Específicamente, en América Latina representan el $30 \%$ de la población total, quienes hoy tienen menos de 35 años, en 2020 representarán el 59 $\%$ de la población del planeta y en 2025 el $75 \%$ de la fuerza laboral mundial, según estudios del Organismo Internacional de Juventud (OIJ, 2016). Por esta razón, el mundo organizacional debe estar preparado para el relevo generacional a partir de las herramientas de transformación que pueda brindar cada institución, incluyendo el Ejército Nacional de Colombia (Correa, Reyes \& Reyes, 2019).

Un insumo importante realizar este estudio es el análisis de las diferentes causales de retiro reportadas en los grados de subteniente, teniente y capitán del Ejército Nacional. En el caso de subteniente, entre los motivos de retiro más frecuentes se encuentran los factores vocacionales, dentro de los cuales están las falsas expectativas, perfiles inadecuados para el cargo, problemas de adaptación a la vida militar, etc. El segundo aspecto es el monetario, dentro del cual se encuentran elementos como problemas de dinero, baja remuneración, entre otras. Por último, 
están los incentivos, como la falta de reconocimiento al trabajo, el incumplimiento de los planes de moral bienestar e inequidad en la asignación de estímulos.

En el grado de teniente existe una dualidad en relación con la primera causal motivante del retiro voluntario, ya que los elementos asociados al ámbito personal y al familiar tienen similares proporciones, seguidos por aspectos monetarios y, por último, aspectos relacionados con el ámbito laboral, dentro de los que se destacan elementos como falta de respaldo, problemas jurídicos, así como fatiga laboral (Comando de Personal [Coper], 2015).

Finalmente, entre las razones para decidir el retiro voluntario en el grado de capitán se encuentran, en primer lugar, elementos relacionados con el ámbito familiar, la enfermedad y la búsqueda de estabilidad familiar. En segundo lugar, motivantes laborales, tales como fatiga laboral, falta de respaldo institucional e inequidad en los traslados. En tercer lugar, motivantes personales ligados principalmente a un nuevo proyecto de vida. (Coper, 2015).

Frente a esta problemática, este trabajo plantea la siguiente pregunta de investigación: ¿qué factores asociados a la retención del personal debe tener en cuenta el Ejército Nacional para disminuir las solicitudes de retiro prematuras de la población millennial?

\section{Marco teórico y conceptual}

Según Center (2014), la generación considerada como millennial ha vivido el recorte del Estado de bienestar y la crisis económica, y ha experimentado sus principales efectos, como el desempleo y la precarización del trabajo. Esta situación lleva a que estos jóvenes busquen fuentes de empleo estables y se incorporen al Ejército Nacional para desarrollar su proyecto vital y profesional, que deberán aplicar a las actividades que se ejercen en el campo militar. De esta manera hay una población millennial altamente calificada lista para ingresar debidamente a las filas en pro de su reconocimiento, canalización y potenciación dentro de la organización, por lo cual es necesario comprender su motivación laboral. Según Robbins y Judge (2009), esta se entiende como la voluntad de ejercer altos niveles de esfuerzo hacia objetivos organizacionales, condicionados por la satisfacción de alguna necesidad individual. Necesidad abordada como un estado interno de cada persona que hace que elija realizar diversas acciones predeterminadas a sus propios intereses.

Es evidente entonces que si el personal no es feliz (satisfecho) con el rol militar que ejerce por falta de reconocimiento de sus potencialidades o por insuficientes compensaciones y garantías organizacionales de carácter monetario, es muy probable 
que abandone su labor. La felicidad enfocada en el trabajo es un aspecto primordial para definir el éxito de una organización porque, según Bejarano (2013), cuando el personal se siente feliz, no escatima esfuerzos en beneficio mutuo; sin embargo, aclara, esta dependerá de cómo las personas gestionan sus propias emociones y las del entorno. Por lo tanto es necesario crear estrategias de prevención en las que se haga rotación de personal y que anticipen la situación del retiro para que no continúe la fuga de capital humano e intelectual potencialmente valioso para la organización.

En articulación con el concepto de felicidad, y su carácter determinante para el éxito de la gestión humana, es fundamental traer a colación la discusión sobre las estrategias disponibles para satisfacer al empleado o trabajador. En este sentido, es necesario establecer qué tipo de contrato psicológico se puede evidenciar en la población millennial que forma parte del Ejército Nacional. En primer lugar, el concepto de contrato psicológico se entiende como las obligaciones mutuas entre empleador y empleado sobre las expectativas que cada uno tiene de su labor y sobre su reciprocidad (Shein, 1980; Argyris, 1960; citados en Tena, 2002). Para efectos de este trabajo, el contrato psicológico se aborda desde la perspectiva de los efectivos, con lo cual es posible hacer un diagnóstico como primer paso para futuras medidas del Ejército.

\section{Metodología}

La información que se analiza en este trabajo es tomada del estudio de Correa et al. (2019), en el cual se caracterizan los factores asociados a la retención del personal que debe tener en cuenta el Ejército Nacional para disminuir los índices de retiro voluntario de la población millennial.

La investigación en mención se desarrolló con un marco metodológico mixto (Sampieri, Fernández \& Baptista, 2018), de orden histórico hermenéutico, que busca tomar lo mejor de cada enfoque "cuanti-cualitativo" para construir una metodología fuerte en términos de indagación, análisis e impacto, de manera que se deduzcan las potenciales debilidades de cada uno cuando se abordan por separado. Así se fortalece el impacto y se aumenta el campo de acción del equipo investigador en pro de la intervención del fenómeno de estudio.

Además, a través de un análisis de casos tipo se dio a la investigación un enfoque inductivo, con el objetivo de afirmar un universal partiendo de casos particulares (Correa et al., 2019). El nivel de conocimiento obtenido fue de tipo exploratorio y descriptivo, considerando que antes de la mencionada investigación 
no existía literatura respecto de la caracterización de la población millennial en las Fuerzas Militares.

\section{Participantes}

De acuerdo con cifras del Coper, a septiembre de 2018 el Ejército Nacional contaba con un total de 118.977 efectivos, distribuidos entre los rangos de oficiales (9.563), suboficiales (32.240) y soldados profesionales (77.174). De esta población, el género masculino es predominante: por rangos representa el $91 \%$ sobre el total de oficiales, $98 \%$ para los suboficiales y $100 \%$ para soldados profesionales.

En el estudio se abordó exclusivamente a la población de oficiales, la cual se subdivide en oficiales del cuerpo administrativo, del cuerpo logístico y de las armas. Consecuente con el papel directo que asumirán los oficiales como comandantes de Batallón y Unidades Operativas Mayores, se eligió a los oficiales de las armas como población objetivo, la cual a septiembre de 2018 contaba con 5.527 efectivos entre subtenientes, tenientes y capitanes.

Se solicitó apoyo a la Escuela Militar de Cadetes "General José María Córdova” (ESMIC) y al Centro de Educación Militar (CEMIL) para disponer del personal de oficiales de las armas en proceso de formación que aplicaran voluntariamente el instrumento diseñado en el estudio. Asimismo, se solicitó a la Dirección de Sanidad (DISAN) apoyo para aplicar en todo el país el instrumento de diagnóstico con el acompañamiento de los psicólogos ubicados en cada una de las unidades.

De este ejercicio se obtuvo una participación nacional de 209 personas, entre alféreces, subtenientes, tenientes y capitanes, con rango de edad entre los 19 y 34 años. De la representación por género, se obtuvo una participación de 182 hombres (87\%) y 27 mujeres (13\%).

Respecto de la representación por armas del personal que formó parte del estudio, 92 personas pertenecen al arma de Infantería (44\%), 40 al arma de Inteligencia (19\%), 18 al Cuerpo Logístico (9 \%) 18 al arma de Ingenieros (9\%), 12 al arma de Caballería (6\%), 8 al arma de comunicaciones (4\%), 9 a artillería (4\%), 9 a Aviación ( 4\%) y 1 participante perteneciente a las Fuerzas Especiales (0,01 \%).

\section{Instrumentos de recolección y de análisis de la información}

En primer lugar, se realizó una revisión documental de la estadística existente sobre la problemática de retiros voluntarios en el Ejército Nacional, de acuerdo con información consolidada por el Comando de Personal (Coper) y la caracterización demográfica de la población objetivo disponible en la plataforma Siath. 
Posteriormente, se diseñó un instrumento de encuesta semiestructurada con el objetivo de indagar los factores motivacionales y detonantes del retiro voluntario (Sampieri et al., 2018). Para diseñar el instrumento se definieron las siguientes categorías: la principal fue el tema de la retención, como transversal se estableció la caracterización del millennial y como subcategorías el proyecto de vida y el contrato psicológico. Acorde con estas categorías se creó el instrumento diagnostico con 32 preguntas de tipo: globales, de opinión, de conocimientos, sensitivas, de antecedentes, de simulación y demográficas (Sampieri et al., 2018).

El sistema de respuestas fue igualmente diseñado para reducir la especulación subjetiva sin perder el carácter semiestructural y de enfoque mixto. Básicamente los criterios de respuesta son: "Selección múltiple, escalas Likert, pregunta abierta y cerrada", los cuales indagan sobre el nivel de aprobación o desaprobación con su respectiva justificación (Correa et al. 2019).

En la etapa de análisis de resultados, se diseñó un matriz que permite detectar los códigos de cada una de las respuestas de los participantes, lo cual permite priorizar e indagar sobre variables centrales fuertemente respaldadas y ya previstas por el equipo investigador, como también detectar nuevas categorías emergentes. Este proceso, denominado saturación teórica, fortalece el proceso de recolección de información hasta que ya no surjan nuevas evidencias o sucesos (Giménez, 2007).

Asimismo, la matriz permite hacer un análisis de tendencias de las respuestas, de las cuales se desprenden tres tipos de categorías que serán asignadas según sea el caso por el equipo investigador: 1) Positiva, si su respuesta estuvo de acuerdo y argumentó exaltando y reconociendo de manera satisfactoria la pregunta. 2) Neutra, si la naturaleza de la respuesta se mantuvo en una posición estable y no presentó ninguna objeción ni satisfacción frente a la situación enfrentada en la pregunta. 3) Negativa, respuesta de carácter polémico que manifiesta descontento e insatisfacción. Lo anterior le da paso finalmente a la contabilización numérica de la frecuencia de las tendencias identificadas en cada una de las preguntas.

\section{Resultados}

De acuerdo con el orden establecido en el instrumento de recolección de información, se delimitaron en primer lugar los aspectos sociodemográficos de la muestra poblacional, considerando variables como edad, sexo, nivel educativo, arma, procedencia, entre otros. En segundo lugar se abordarán los resultados de las subcategorías de acuerdo con el orden establecido, es decir, primero el proyecto de vida y luego el contrato psicológico. 


\section{Características sociodemográficas}

Para la caracterización sociodemográfica del militar oficial millennial se establecieron doce preguntas cerradas que indagaban: (1) edad, (2) género, (3) estrato, (4) grado, (5) arma, (6) procedencia, (7) estado civil, (8) nivel de educación, (9) hijos, (10) cantidad de hijos, (11) esposo y/o compañero sentimental y (12) afinidad compañero sentimental con FF. MM.

Como resultado de lo anterior se identifica al militar oficial millennial de la siguiente forma: son mayoritariamente hombres, con un promedio de edad de 27 años y estrato socioeconómico nivel tres (3), procedentes de la región Andina, de estado civil solteros, sin hijos, y con nivel de educación de pregrado (figuras 1, 2, 3 y 4).

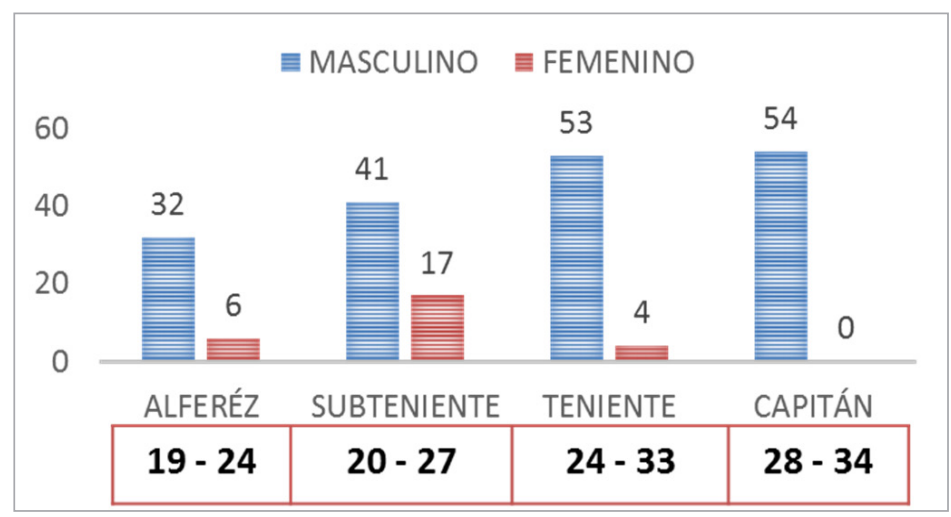

Figura 1. Relación por grado, sexo y edad.

Fuente: Correa et al. (2019).

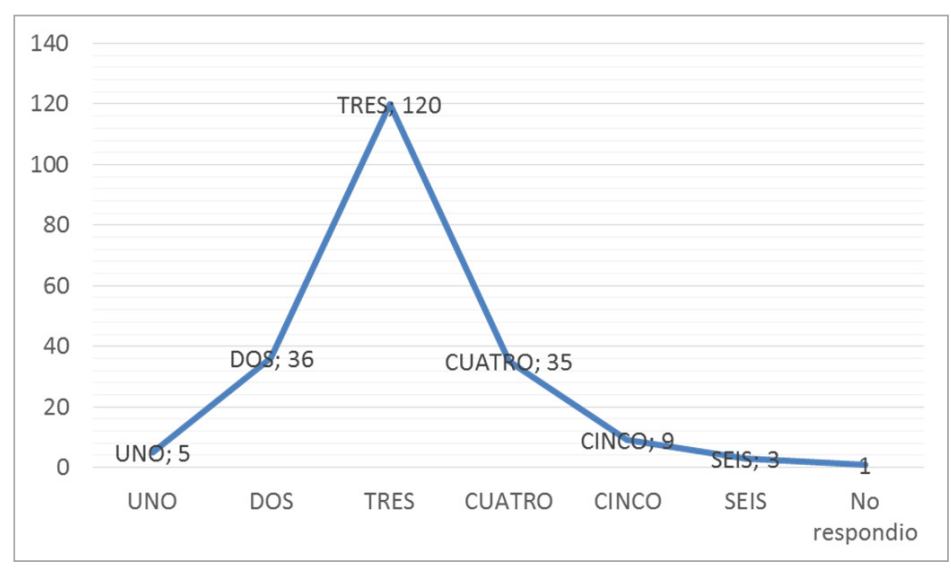

Figura 2. Estrato socioeconómico.

Fuente: Correa et al. (2019). 


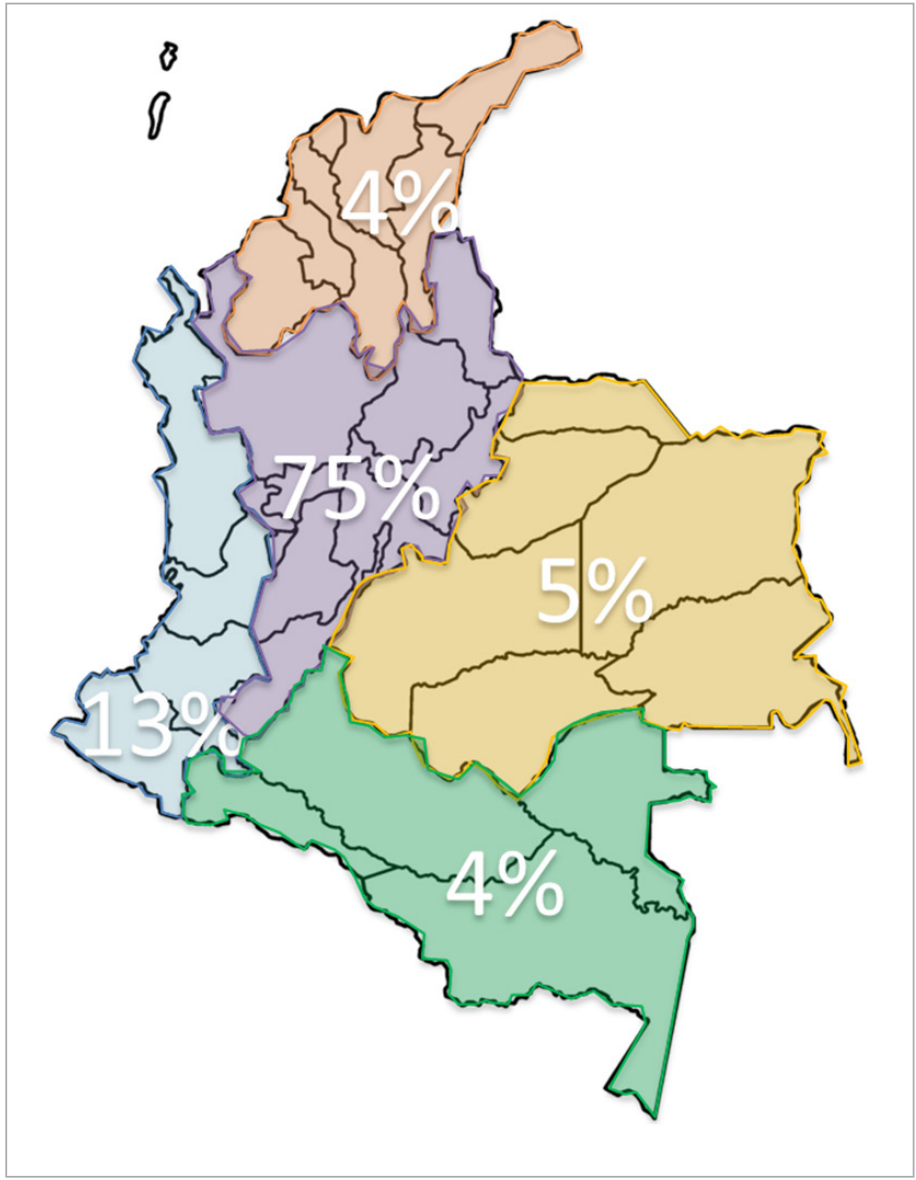

Figura 3. Lugar de procedencia.

Fuente: Correa et al. (2019).

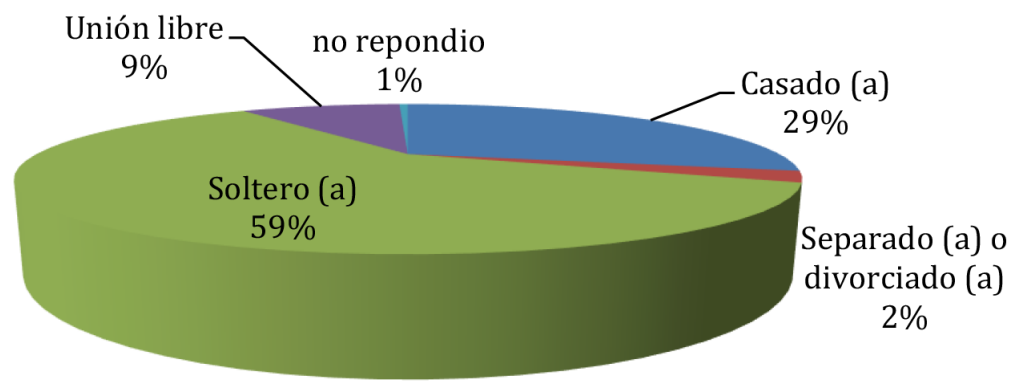

Figura 4. Estado civil.

Fuente: Correa et al. (2019). 


\section{Proyecto de vida}

Para definir esta categoría se tomó como referencia a Zauza (2010), quien subdivide el proyecto de vida en cuatro categorías: (1) ámbito profesional: expectativas y planes de estudio y procesos de formación; (2) ámbito familiar: relación que tiene con el actual y futuro núcleo familiar; (3) ámbito financiero: aspiraciones pecuniarias y de capital inmobiliario, y (4) ámbito social: relaciones e imagen que tiene el individuo para con la sociedad.

De acuerdo con lo anterior, se establecieron diez preguntas con respuestas de tipo cerradas y escala Likert, en las cuales a algunos casos se les solicitó dar una justificación con el objetivo de identificar la proyección personal y profesional del oficial militar millennial en el mediano y largo plazo.

\section{ÁMBITO PROFESIONAL}

De esta indagación se puede especificar lo siguiente: el $90 \%$ de la población expresa el deseo de emprender una formación profesional complementaria en los próximos diez años, principalmente encaminada a las áreas de administración, derecho, relaciones internacionales e ingenierías (figura 5).

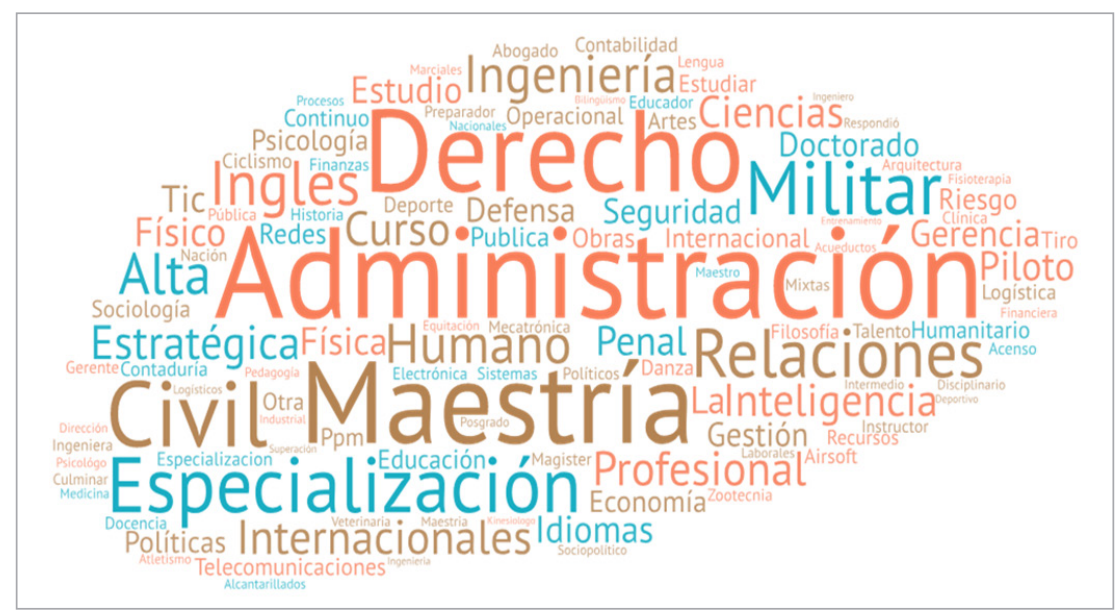

Figura 5. Representación de las áreas de interés para formación complementaria. Fuente: Correa et al. (2019).

Respecto de las posibles limitantes para materializar el plan complementario de estudios, se formuló la siguiente pregunta: “¿Cree usted que el Ejército Nacional le brinda a su personal posibilidades para ejecutarlo (desarrollo profesional) a 
mediano (2 y 5 años) y largo plazo (10 ańos)?”. La respuesta del $51 \%$ de la población fue positiva, frente al $49 \%$ que eligió la opción negativa.

Ante este "empate técnico" se decidió analizar las tendencias positivas y negativas que se obtuvieron en la justificación de esta pregunta y se obtuvo los siguientes resultados: (1) las respuestas positivas recalcan la importancia de profesionalizar la fuerza impulsada por los comandantes (superiores inmediatos); (2) las respuestas negativas mencionan factores en contra: falta de tiempo por carga laboral, falta de voluntad por parte de los comandantes, influencias y procesos de selección centrados en personal adscrito a unidades militares en ciudades principales, plan de traslados desarmonizado con los apoyos en procesos de formación profesional y técnica, y baja cobertura en programas de formación virtual.

\section{Ámbito FAMILIAR}

En esta subcategoría del proyecto de vida se indagaron factores como la favorabilidad en los cambios familiares debido a pertenecer al Ejército Nacional, y el nivel de proporción entre las compensaciones recibidas frente a los cambios familiares surgidos por pertenecer a la fuerza (figuras 6 y 7 ).

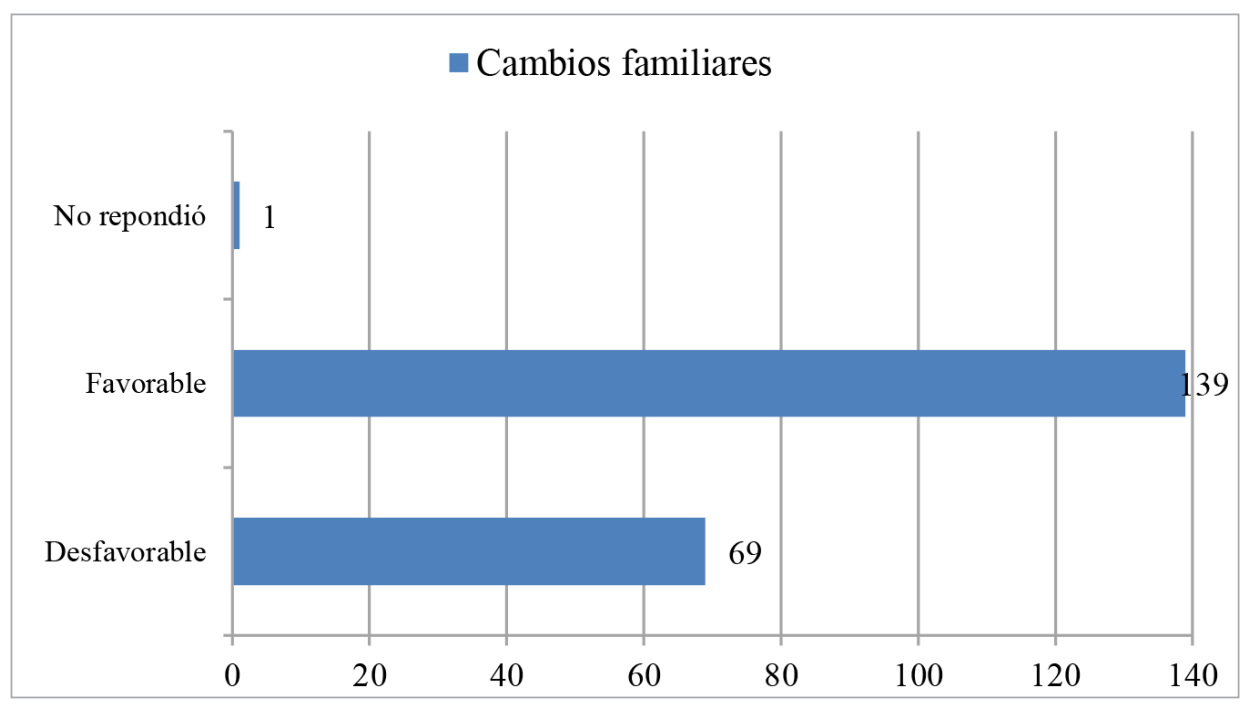

Figura 6. Clasificación de los cambios que han vivido los participantes en sus relaciones familiares por ser parte de una organización como el Ejército Nacional.

Fuente: Correa et al. (2019). 


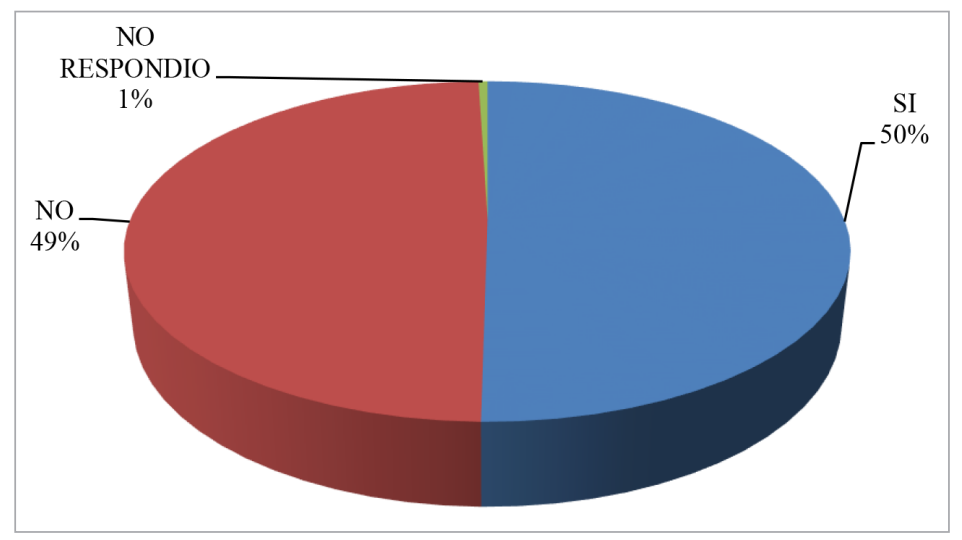

Figura 7. ¿Cree usted que las compensaciones (económicas de bienestar y de estatus social) han sido proporcionales con los cambios familiares que ha vivido?

Fuente: Correa et al. (2019).

Ante el mínimo margen de diferencia que se observa en la figura 7, se realizó un análisis de tendencias positivas y negativas y se obtuvieron los siguientes resultados: (1) respuestas positivas respecto del sistema de compensaciones y beneficios para los militares: primas, acceso a clubs, subsidios de vivienda, entre otros. (2) Respuestas negativas referentes a insuficientes compensaciones como salarios y primas para costear gastos familiares asociados a traslados, al igual que baja cobertura en los planes de moral y bienestar para poder disfrutar en familia.

\section{ÁMBITO FINANCIERO}

Para definir las aspiraciones pecuniarias y de capital inmobiliario se establecieron tres preguntas: (1) ¿ ¿Su trabajo en el Ejército Nacional es su principal fuente de ingresos? (2) ¿Tiene como objetivo iniciar algún proyecto laboral paralelo a su profesión en el Ejército Nacional? (3) Basado en su experiencia frente a las prestaciones salariales, sociales y el plan de bienestar que le brinda el Ejército Nacional, seleccione su clasificación: excelente, buena, regular, pésima. En este ítem se encontró que el $93 \%$ del personal encuestado expresó que su trabajo en el Ejército Nacional es su única fuente de ingresos, mientras que el $65 \%$ se proyecta a tener un proyecto paralelo a su carrera militar (figuras 8 y 9 ).

Como observa en la figura 9, la percepción frente a las prestaciones sociales y los planes de bienestar que recibe el militar oficial millennial es buena (47\%) y excelente $(11 \%)$. La tendencia de estas respuestas positivas se basa en la percepción favorable frente a la puntualidad en el pago de salarios básicos y primas, además de que consideran como justas dichas remuneraciones para el sustento personal y familiar. 


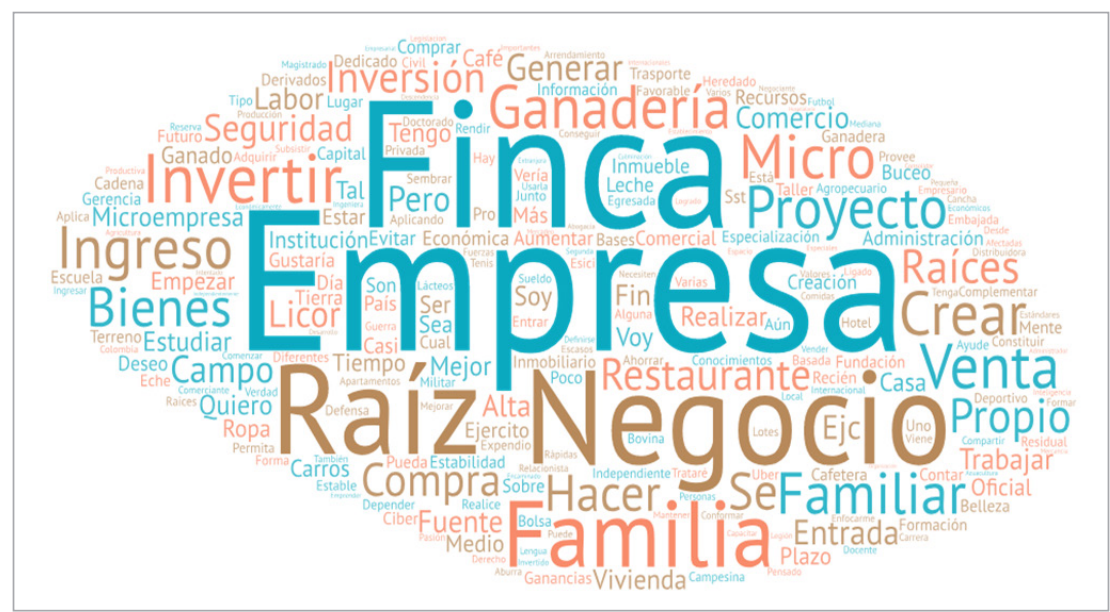

Figura 8. Tendencia del proyecto laboral paralelo al Ejército Nacional. Fuente: Correa et al. (2019).

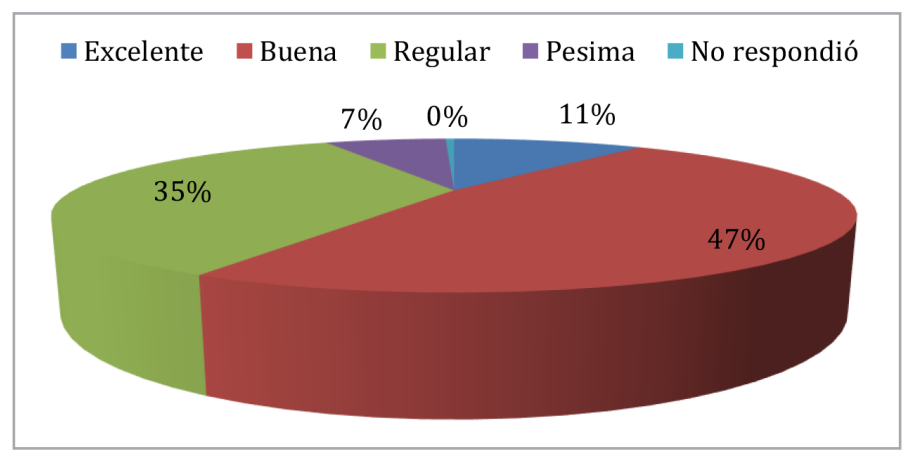

Figura 9. Distribución de la muestra según percepción de las prestaciones sociales y planes de bienestar.

Fuente: Correa et al. (2019).

\section{ÁmBito social}

La última subcategoría para caracterizar el proyecto de vida se compone del prestigio y la posición que el individuo tiene y / o quiere conseguir. Para abordar el factor del prestigio se planteó esta pregunta: “¿Cuál cree que es la percepción de la población civil frente al Ejército Nacional y al militar?”, frente a la cual se obtuvo una tendencia mayoritariamente positiva, sustentada en conceptos como honor, orgullo, familia, disciplina, lealtad, vocación y sacrificio, en cuanto fueron las palabras repetidas en la justificación de esta respuesta (figura 10). 


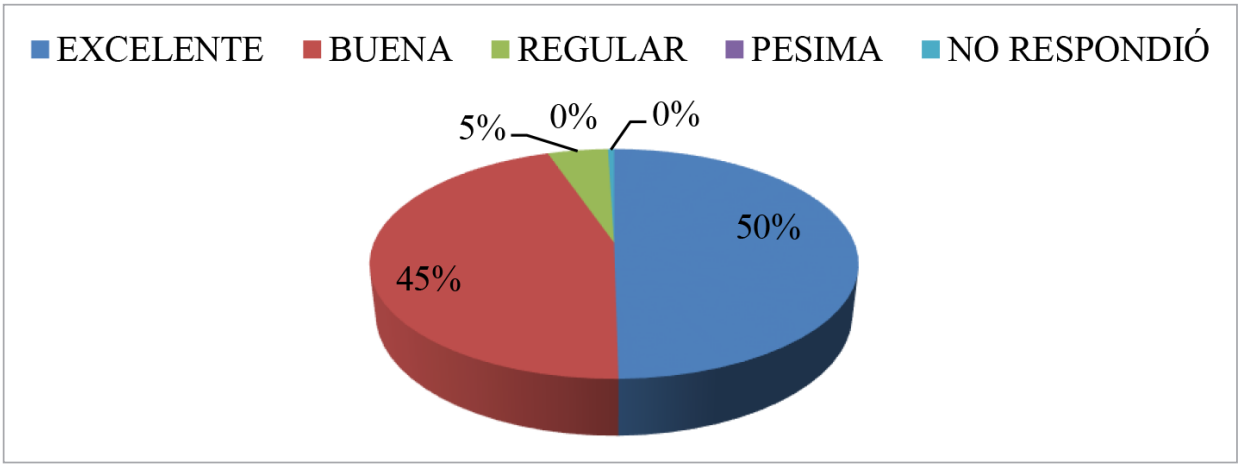

Figura 10. ¿Cuál cree que es la percepción de la población civil frente al Ejército Nacional y al militar?

Fuente: Correa et al. (2019).

En cuanto a la percepción personal que tiene el oficial militar millennial respecto de pertenecer al Ejército Nacional, el $99 \%$ respondió que siente honor y orgullo formar parte de la institución (figura 11).

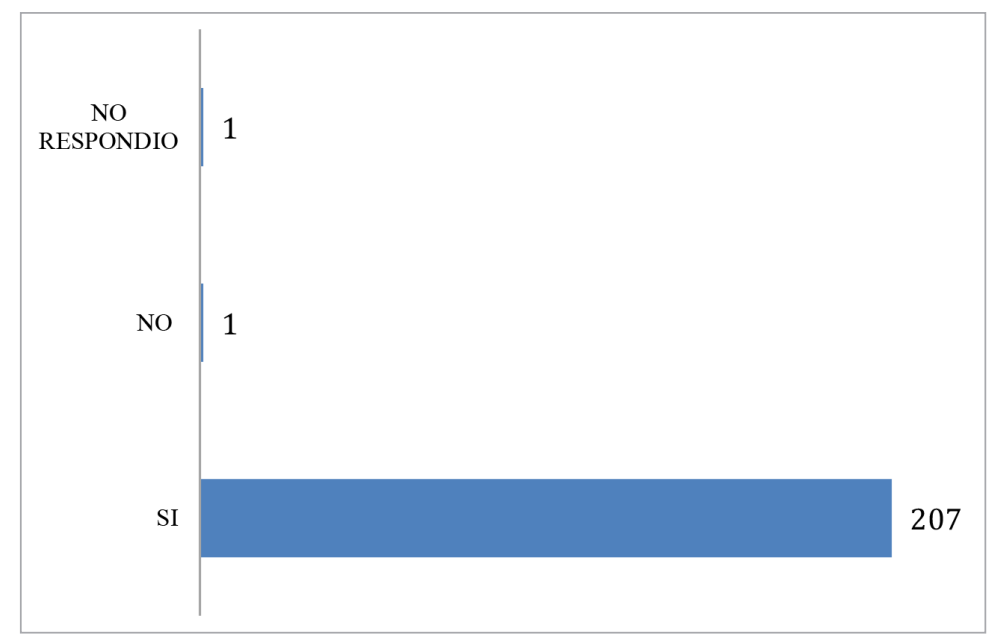

Figura 11. ¿¿e genera honor y orgullo formar parte del Ejército Nacional? Fuente: Correa et al. (2019).

Finalmente, y con el fin de confirmar cuál de los ámbitos abordados en el proyecto de vida del oficial militar millennial perciben como más importante, se preguntó explícitamente lo siguiente cuál es el ámbito más importante (figura 12). 


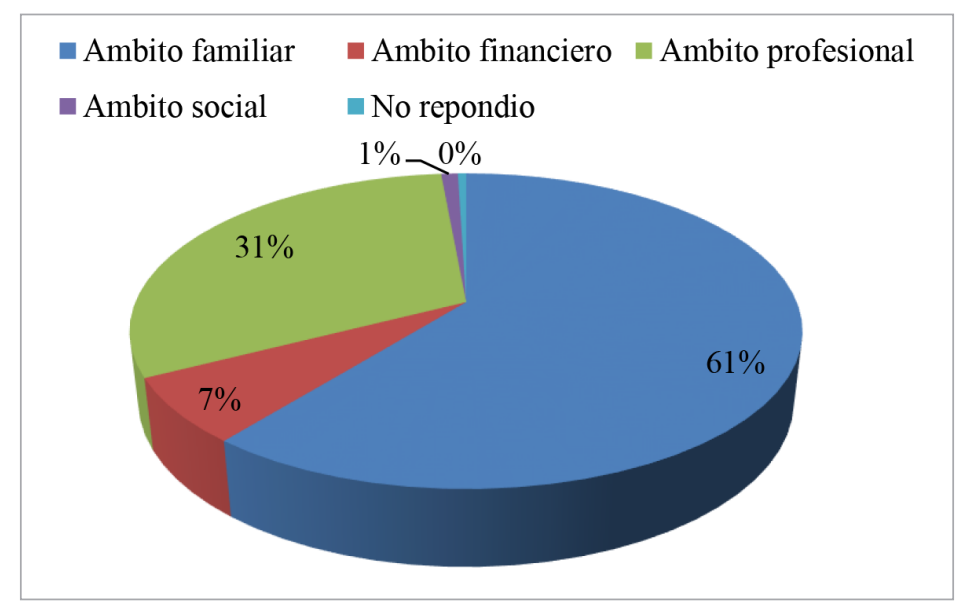

Figura 12. Ámbito más importante para el oficial militar millennial. Fuente: Correa et al. (2019).

\section{Contrato psicológico}

Según Núñez (2013), el contrato psicológico se compone de tres factores: (1) Percepción institucional: construcción que es "propiedad" de los públicos usuarios. (2) Justicia: grado de equidad que el individuo percibe en la organización. (3) Confianza: grado en que el individuo está dispuesto a asumir situaciones de vulnerabilidad.

Para abordar estos tres factores, el instrumento de diagnóstico incluyó catorce preguntas con respuestas de tipo cerradas y escala Likert, incluyendo la respectiva justificación, con el propósito de comprender la relación que tiene el oficial militar millennial con la institución.

\section{Percepción institucional}

El primer factor del contrato psicológico se abordó a través de la formulación de cuatro preguntas: (1) en una palabra, describa qué significa el Ejército Nacional como institución para usted. (2) ¿Le recomendaría a un amigo o familiar incorporarse?, ¿por qué? (3) ¿Hasta qué grado se proyecta en la institución?, ¿por qué? (4) Indique de 1 a 4 su nivel de intención actual de realizar retiro voluntario del Ejército Nacional.

Los resultados de las dos primeras preguntas son coherentes con los hallazgos en el ámbito social del proyecto de vida, ya que las palabras que más usaron los participantes para describir el significado de pertenecer al Ejército Nacional son orgullo, honor, disciplina, familia y lealtad (figura 13). Asimismo, el $87 \%$ del personal encuestado le recomendaría a un amigo o familiar incorporarse a la institución, principalmente, por los siguientes aspectos positivos: la estabilidad econó- 
mica y laboral que brinda la institución, y el desarrollo de valores y habilidades en el saber y el hacer, directamente relacionados con la vocación de la carrera militar.

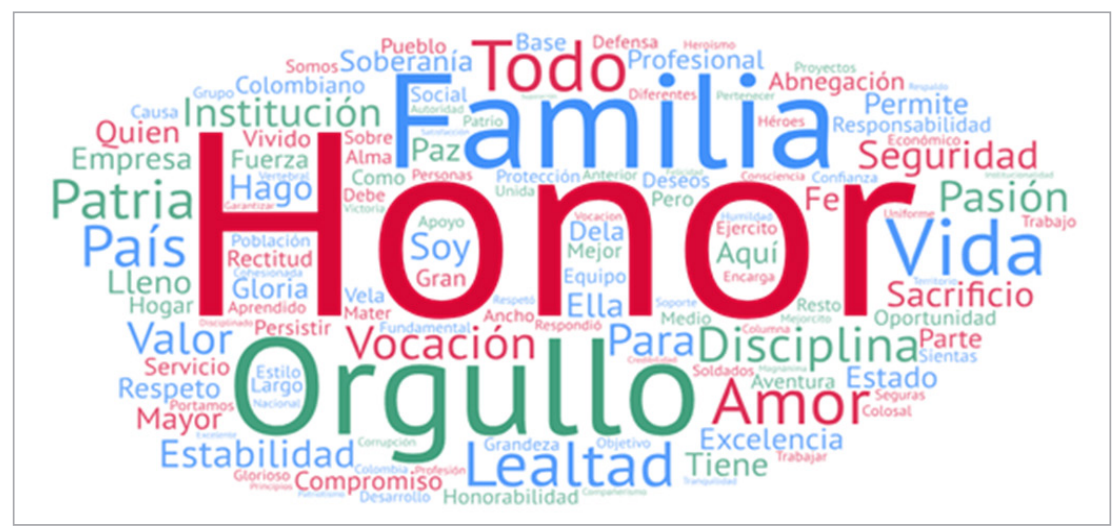

Figura 13. Principales tendencias descriptivas frente al significado del Ejército para el oficial militar millennial.

Fuente: Correa et al. (2019).

Respecto de la proyección del oficial militar millennial en la institución, y la posible intención de solicitar el retiro voluntario, se encontró que la tendencia es mayormente positiva, es decir, un gran porcentaje de la muestra favorece la permanencia a largo plazo en la institución. De acuerdo con las justificaciones obtenidas para ambas respuestas, el militar oficial millennial expresa fuertes intenciones en mostrar habilidades, capacidades y actitudes en pro de mejorar los procesos internos de la institución. Por tanto, el $58 \%$ del personal considera improbable solicitar retiro voluntario (figuras 14 y 15).

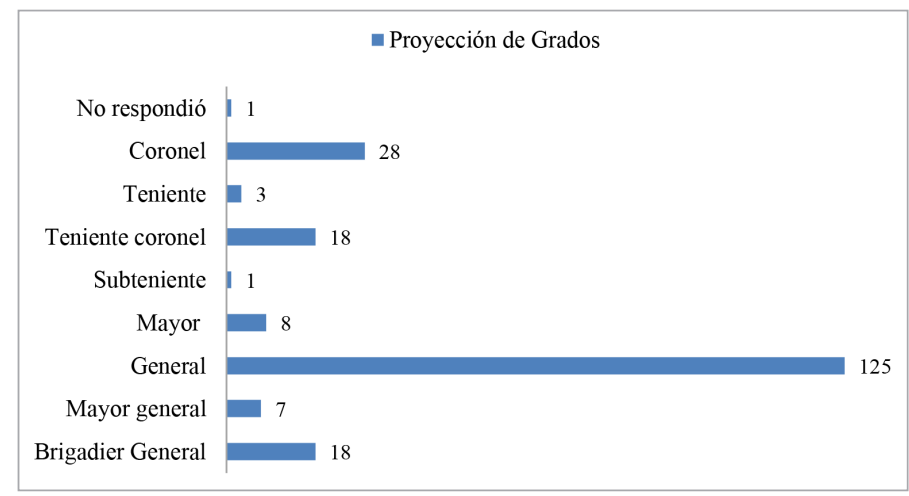

Figura 14. ¿¿Hasta qué grado se proyecta en la institución? Fuente: Correa et al. (2019). 


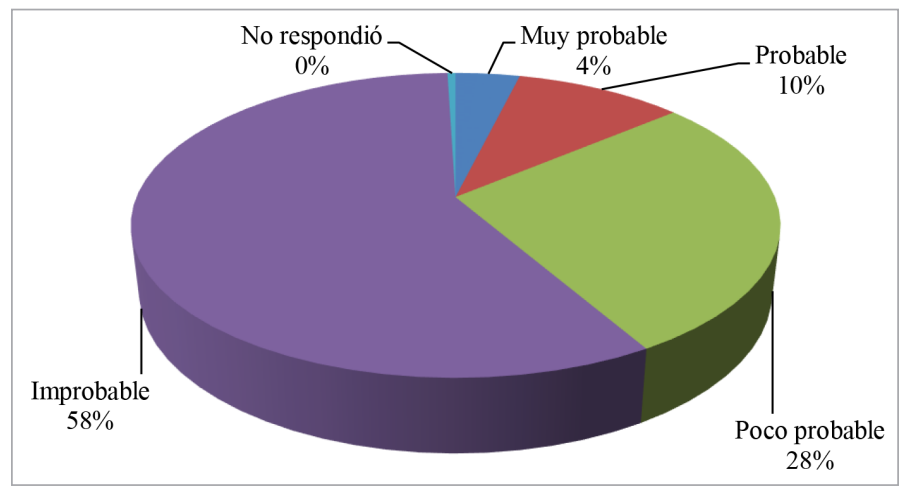

Figura 15. Nivel de intención actual de realizar retiro voluntario del Ejército Nacional. Fuente: Correa et al. (2019).

\section{Justicia}

La segunda subcategoría que compone el contrato psicológico trata del grado de equidad que el individuo percibe en el intercambio de contribuciones y compensaciones con su organización (Gracia, Peiró \& Mañas, 2007). Por lo anterior, y con el fin de delimitar esa percepción de justicia por parte del oficial militar millennial, se formularon dos preguntas: (1) ¿Cree usted que las compensaciones (económicas, de bienestar y de estatus social) han sido proporcionales con las labores que ha desempeñado? (2) ¿Considera que los traslados que se llevan a cabo en el Ejército Nacional se realizan de forma equitativa?, ¿por qué? (figuras 16 y 17).

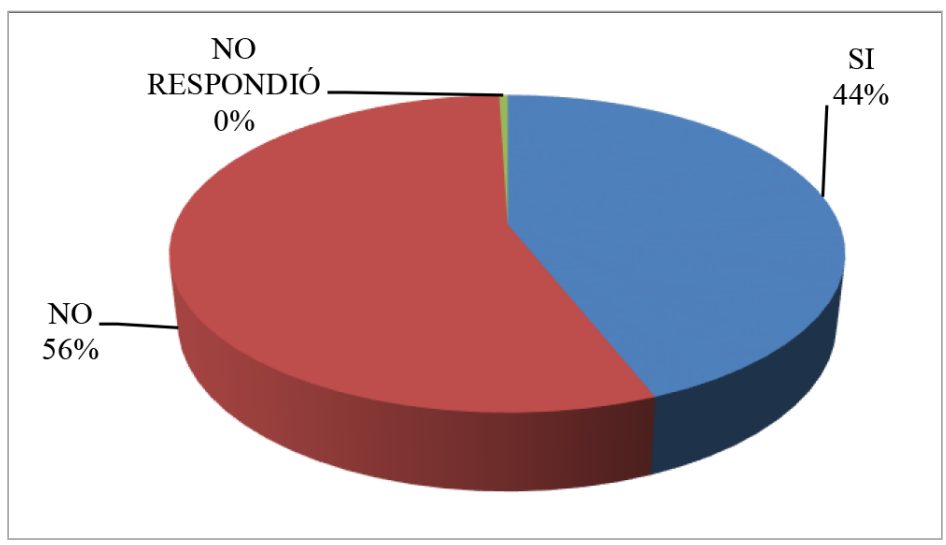

Figura 16. ¿Cree usted que las compensaciones (económicas, de bienestar y de estatus social) han sido proporcionales con las labores que ha desempeñado? Fuente: Correa et al. (2019). 


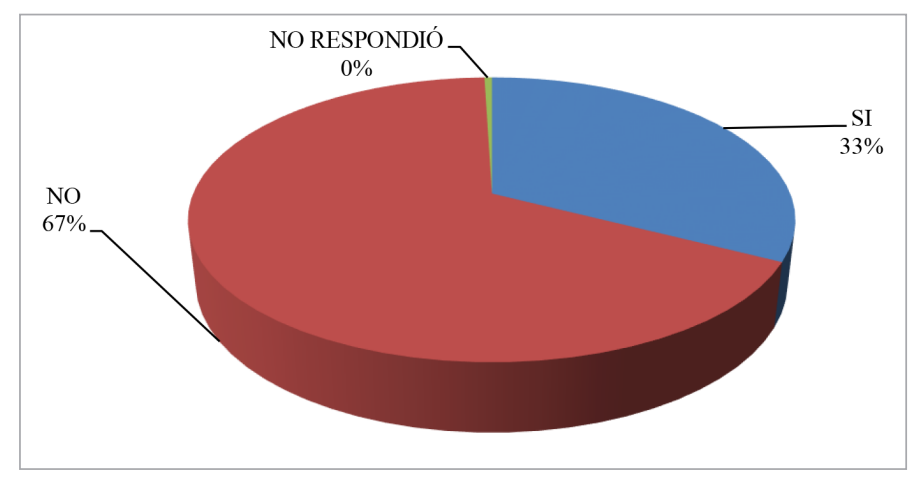

Figura 17. ¿Considera que los traslados que se llevan a cabo en el Ejército Nacional se realizan de forma equitativa?, ¿por qué?

Fuente: Correa et al. (2019).

La tendencia negativa para las preguntas de la subcategoría justicia se basan en la inconformidad de las compensaciones recibidas frente a los siguientes factores: labores desempeñadas, responsabilidad, sacrificio, riesgo constante y disponibilidad 24 horas.

Igualmente, respecto de la percepción negativa frente a la forma como se llevan a cabo los traslados, el militar oficial millennial expresa que a pesar de existir en la institución plataformas como el MOCE, que han regulado en cierta medida la asignación de traslados por conveniencia, aún se siguen presentando situaciones como influencias, padrinos, recomendados, lazos de consanguinidad, corrupción, entre otros.

\section{Confianza}

Esta subcategoría hace referencia al grado en que el individuo está dispuesto a asumir condiciones de vulnerabilidad sobre el supuesto de que la organización, la dirección de esta o su superior inmediato no harán un uso perjudicial para él (Gracia et al. 2007).

El abordaje del factor confianza se realizó a través de siete preguntas: (1) Indique de 1 a 4 su nivel de satisfacción con la relación que actualmente tiene con su superior inmediato. (2) Indique de 1 a 4 su nivel de satisfacción frente al grado de reconocimiento que tiene la institución hacia sus logros y conocimientos, ¿por qué? (3) ¿El ejercicio militar le ha implicado daños físicos y/o psicológicos (diagnosticados por junta médica laboral)?, ¿̨cuáles? (4) Indique de 1 a 4 su nivel de satisfacción propia y familiar frente al servicio de sanidad de la institución?, ¿por qué? (5) ¿La institución le brinda los recursos necesarios para el desarrollo óptimo de sus funciones y/o objetivos que debe alcanzar en su actividad como militar?, ¿por qué? (6) ¿Cree usted que la doctrina militar aporta positivamente a su estilo de vida?, 
¿por qué? (7) ¿El Ejército Nacional le ha aportado herramientas para desarrollar su proyecto de vida?, ¿por qué?

La tendencia positiva evidenciada que el $80 \%$ de la muestra expresa sentirse satisfecho ( $45 \%$ ) y muy satisfecho (35\%), lo cual se sustenta en factores percibidos por parte del personal encuestado como: respeto, buen trato, equidad, toma de decisiones adecuadas, genera confianza, es justo, planea, orienta, corrige de forma adecuada, entre otros comentarios (figura 18).

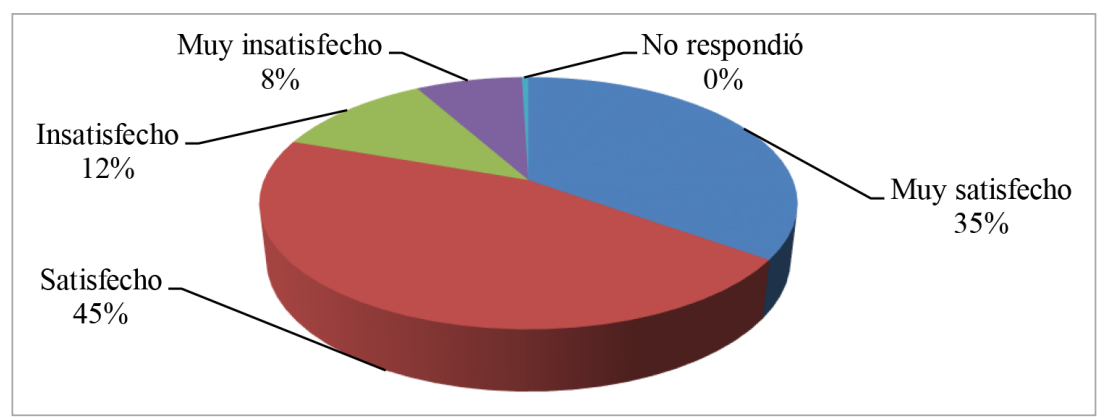

Figura 18. Nivel de satisfacción con la relación que actualmente tiene con su superior inmediato. Fuente: Correa et al. (2019).

El nivel de satisfacción respecto del reconocimiento hacia logros y conocimientos demuestra una tendencia positiva, ya que el oficial militar millennial tiene la conciencia de que estos le son otorgados en la medida en que se demuestre el esfuerzo, la dedicación y la disciplina en las tareas asignadas. La demostración de estos resultados le permite al millennial acceder a procesos de capacitación y formación, lo cual constituye un factor motivante propio de esta generación (figura 19).

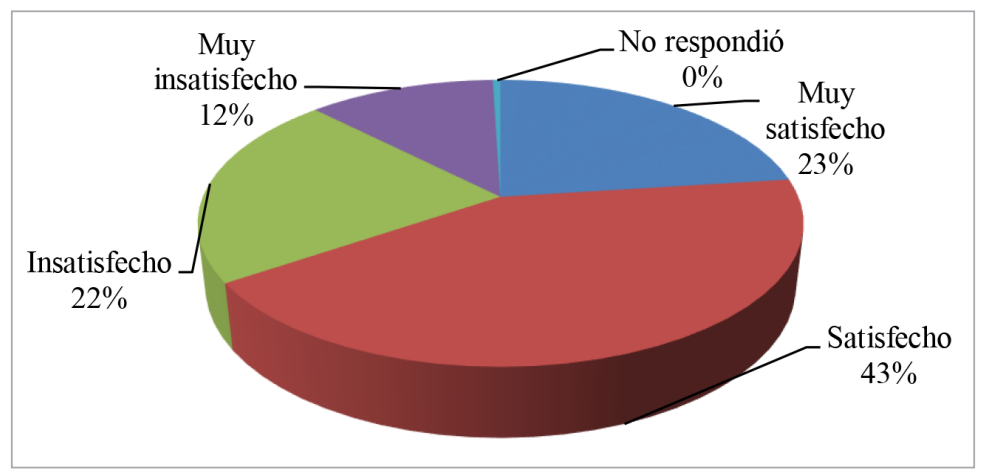

Figura 19. Grado de reconocimiento que tiene la institución hacia sus logros y conocimientos. Justificación.

Fuente: Correa et al. (2019). 
El resultado frente al nivel de satisfacción de los servicios de sanidad es ajustado, aunque con prevalencia positiva, como se muestra en la figura 20. Las justificaciones que respaldan la tendencia positiva expresan, en su mayoría, una conformidad con el sistema de sanidad del Ejército, el cual, aunque reconocen que tiene fallas, es superior en calidad frente al sistema general de salud en Colombia, el cual se encuentra en crisis.

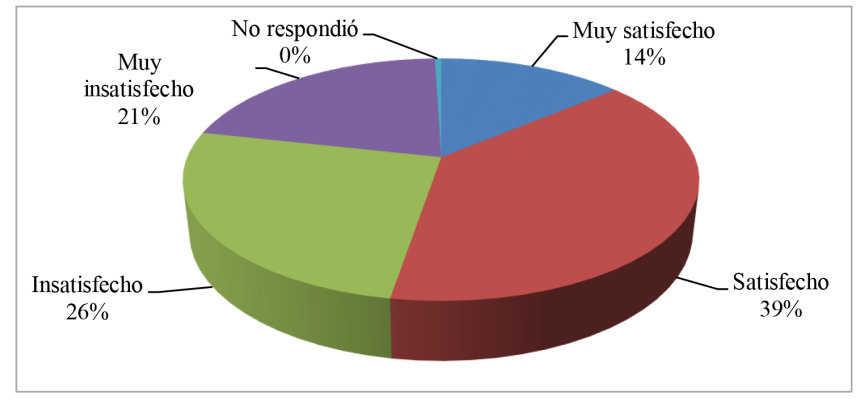

Figura 20. Nivel de satisfacción propia y familiar frente al servicio de sanidad de la Institución. Fuente: Correa et al. (2019).

Frente al análisis de la tendencia negativa, el personal expresa haber encontrado varias inconsistencias con el sistema de sanidad de la institución: demora en las citas, más tramites, menos medicamentos, mala atención a los usuarios, difícil acceso a especialistas, entre otras.

Respecto de la justificación que aportan los oficiales militares millennial se expresan aspectos positivos frente a la institución, considerando que está "intenta distribuir los recursos de forma adecuada para brindar a las unidades los medios y herramientas necesarias para cumplir las misiones asignadas" (figura 21).

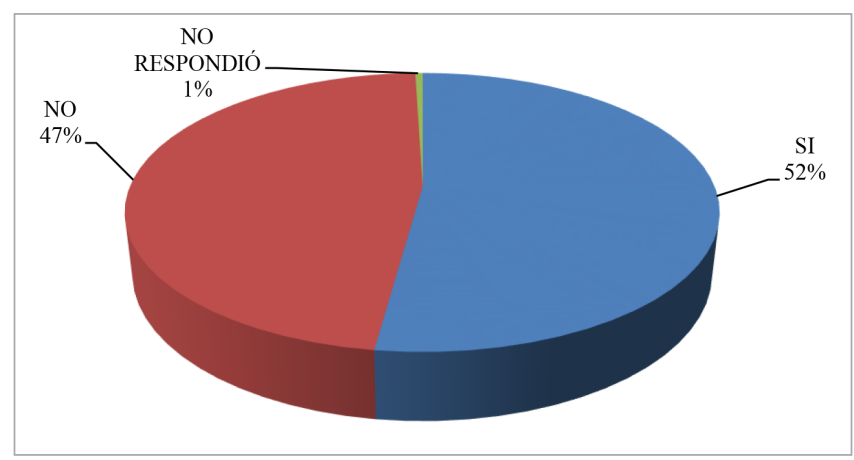

Figura 21. Recursos necesarios para el desarrollo óptimo de sus funciones y/o objetivos que debe alcanzar en su actividad como militar.

Fuente: Correa et al. (2019). 
Referente al aporte que la doctrina militar ha significado en el estilo de vida del oficial militar millennial, el $93 \%$ del personal expresa una tendencia positiva y argumenta que gracias a la doctrina militar ha "generado hábitos y principios de disciplina, responsabilidad, optimizar tareas y tiempo, honestidad, orden, integridad, visión optimista hacia el futuro, buen comportamiento como ciudadanos y administración del talento humano" (figura 22).

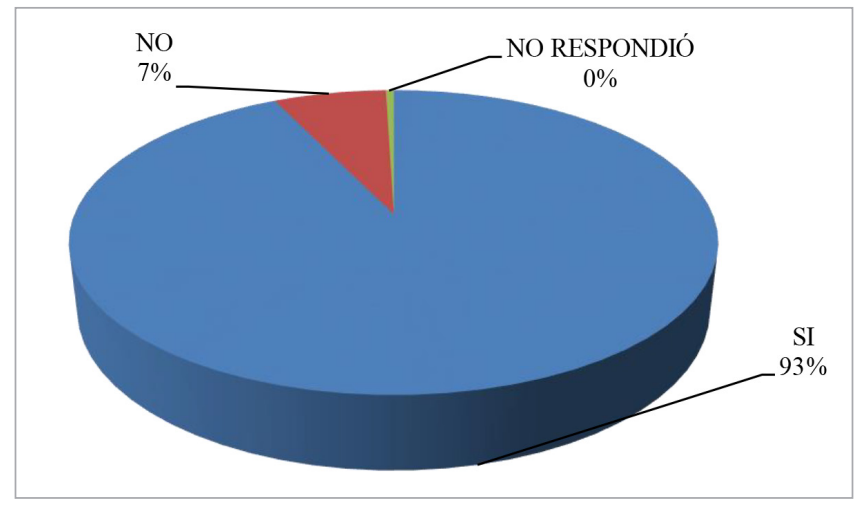

Figura 22. ¿Cree usted que la doctrina militar aporta positivamente a su estilo de vida? Fuente: Correa et al. (2019).

En los oficiales militares millennial que dieron respuestas orientadas a tendencias positivas se observa que las herramientas que les brinda el Ejército Nacional para desarrollar su proyecto de vida están basadas en estabilidad económica y laboral; aprendizaje en valores para la vida personal como militar, capacitación, viajes, ahorro para vivienda, conocimiento, experiencia, subsidios familiares, capacidades humanas, capacitación en ámbitos operacionales (táctica, técnica y físico) (figura 23).

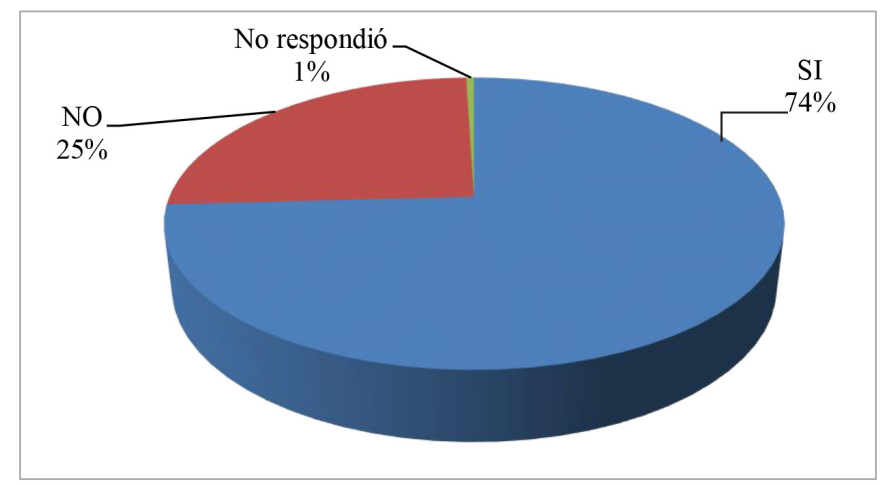

Figura 23. ¿El Ejército Nacional le ha aportado herramientas para desarrollar su proyecto de vida? Fuente: Correa et al. (2019). 


\section{Discusión}

Los resultados del componente demográfico basados en las estadísticas del instrumento de diagnóstico aplicado permite concluir que las características individuales del típico militar oficial millennial demuestran lo siguiente: son más hombres que mujeres, con una edad promedio de 27 años, de un estrato socioeconómico tres, con rango subteniente, un nivel de estudios profesional, perteneciente predominantemente al arma de Infantería, originariamente de la ciudad de Bogotá y se caracterizan por ser solteros sin obligaciones familiares de hijos.

Debido al carácter exploratorio de la investigación, este primer ejercicio de identificación y descripción del millennial inmerso en una organización del sector defensa, perteneciente al ámbito público, representa un potencial de mejoramiento de las prácticas, programas y políticas dirigidas en atención a este segmento generacional. La importancia de esta información radica en que este segmento poblacional va a ser el de mayor presencia en la planta de personal de la fuerza y va a ocupar cargos de gran jerarquía, nivel y autoridad, de manera que es crítica la presencia de fenómenos como el retiro voluntario por los diversos factores identificados anteriormente. Por lo tanto, para el Ejército Nacional constituye una oportunidad para aplicar este instrumento y metodología a una mayor escala y en todo el territorio nacional en términos de número de efectivos, número de armas y otro tipo de generaciones presentes en sus filas.

Por lo tanto, la institución debe tener en cuenta que la tipología militar oficial millennial se caracteriza por fundamentar su proyecto de vida alrededor del ámbito familiar, valora la estabilidad económica que le da la organización y exige un equilibrio entre el cumplimiento de la misión y su esfera personal. Sin embargo, también consideran importante desempeñarse adecuadamente en el ámbito profesional como respuesta a un contexto cada vez más competitivo, así que en contribución exigen tener una estabilidad económica para solventar sus necesidades, así como mantener el estatus y honor que le reviste portar el uniforme del Ejército Nacional.

\section{Conclusiones}

Se concluye que el Ejército Nacional debe tener en cuenta esta caracterización del proyecto de vida del militar oficial millennial con el fin de articular los planes de retención de personal y demás procesos de gestión humana con estrategias que logren: 
1. Equilibrar el ámbito familiar frente al cumplimiento de la misión del militar oficial millennial dentro de la fuerza. Para esto debe continuar priorizando los programas de bienestar al personal de primera línea y efectuando seguimiento a su núcleo familiar.

2. Articular el sistema de educación y doctrina de la fuerza, ampliando su oferta y cobertura a través de medios tecnológicos y alianzas con el mercado educativo que permitan al militar oficial millennial acceder y cumplir con su proyecto de vida en el ámbito profesional.

3. Revisar y evaluar el sistema de compensaciones actual, con el fin de incentivar al militar oficial millennial a cumplir su proyecto de vida en sus diversos ámbitos, adicional al familiar, que es el que predomina actualmente para la fuerza.

4. Fortalecer el sistema de valores y principios de la fuerza, utilizándolos como herramienta de identidad que permita retener al militar oficial millennial. Asimismo, se debe resaltar el estatus que tiene frente a su familia y frente a la sociedad, además de configurar un sistema de responsabilidad social empresarial que motive no solo al personal activo, sino al talento humano potencial a incorporar en futuros cursos administrativos y de armas.

Finalmente, se concluye que para disminuir la deserción de la población militar oficial millennial es necesario tener en cuenta factores asociados a la formación del contrato psicológico. A partir de los resultados del estudio se encuentra que es primordial considerar los siguientes componentes para desarrollar procesos de retención y bienestar:

1. En cuanto al ítem de imagen institucional, se evidencia que existe una percepción favorable en cuanto a sentido de pertenencia, pues esta se encuentra orientada en sentimientos de orgullo y honor de servir a la patria. Esto incide directamente en el desarrollo de valores y habilidades que se encuentran motivados directamente por factores intrínsecos (individuo) y extrínsecos (institución) en el desarrollo del plan de carrera. Aunque es claro para el militar oficial millennial las falencias de la institución, existe una baja intención de retiro voluntario debido a que tienen el interés de ascender para poder transformar los procesos que se consideran mal direccionados.

2. En cuanto al ámbito de justicia, existe un alto grado de inconformidad frente al papel que se ejerce, los beneficios recibidos y el funcionamiento 
interno- externo de la institución (traslados, ascensos, inequidad salarial, riesgo vs beneficios etc.).

3. En el ámbito de confianza, el militar oficial millennial registra un alto grado frente a su institución, por cuanto reconoce que para lograr un adecuado funcionamiento es necesario que haya una mejor distribución de los recursos (manejo de la dotación, logística y dotación tecnológica), reconocimientos de logros, comisiones, acceso a educación, buenos servicios de sanidad y de bienestar. Además, aunque consideran que en ocasiones estos procesos se desvían por actos de corrupción, afirman su interés en mejorar de forma continua para aumentar la calidad de la cobertura nacional.

También se recomienda enfatizar la construcción de planes de retención que tengan en cuenta los factores que se han identificado respecto a la población estudiada, especialmente los tópicos que arrojaron las categorías emergentes: profesionalización, honor, estabilidad laboral, sistemas de compensación, vocación militar, inequidad, jerarquía, servicio, crecimiento laboral y personal, doctrina militar, valores y principios.

Lo anterior permitirá contribuir a disminuir la deserción de la población millennial en el Ejército Nacional. No obstante estos aportes, se recomienda aumentar la muestra para futuros estudios teniendo en cuenta que es una institución con cobertura nacional, de manera que es necesario expandir el instrumento a todas las armas e involucrar al militar millennial suboficial y soldado profesional.

Asimismo, se debe tener en cuenta que la información que se tenga sobre talento humano de forma sistemática, consciente y proactiva podrá optimizar la fuerza de trabajo. Esto implica que el capital intelectual se convierta en un agente indispensable para el presente y el futuro de la organización, por lo cual se deberá considerar la caracterización de los factores asociados a la retención del personal para contribuir con la disminución de la deserción de las otras generaciones que forman parte del funcionamiento de la institución: baby boomers y generación x.

Finalmente, y teniendo en cuenta que actualmente el contexto laboral cambiante exige a las organizaciones crear nuevas formas de funcionamiento y organización del talento humano, es importante estudiar estrategias de atracción que permitan a la institución estar preparada para el relevo generacional. Para cumplir este objetivo es indispensable comprender las características propias de la generación de los centennials, lo cual permitirá generar nuevas estrategias de atracción para la incorporación a la fuerza. 


\section{Agradecimientos}

Esta investigación ha sido realizada gracias al apoyo de la Escuela Militar de Cadetes "General José María Córdova” (ESMIC) y a la Dirección de Investigación, Innovación y Desarrollo de Personal del Ejército Nacional.

\section{Financiación}

Los autores no declaran fuente de financiación para la realización de la presente investigación.

\section{Referencias}

Argyris, C. (1960). Understanding organizational Behavior. Homewood, IL.: Dorsey Press.

Bejarano, P. G. (2013). Gestión del talento humano como estrategia para retención (Trabajo de Especialización en Gestión del Talento Humano). Universidad de Medellín, Facultad de Ciencias Económicas y Administrativas. Medellín, Colombia. Recuperado de https:// repository.udem.edu.co/bitstream/handle/11407/160/Gesti\%C3\%B3n\%20del\%20 talento $\% 20$ humano $\% 20$ como $\% 20$ estrategia $\% 20$ para $\% 20$ retenci $\%$ C3\%B3n $\% 20$ del $\% 20$ personal.pdf?sequence $=1$

Center, P. R. (marzo 7 de 2014). Millennials in adulthood. Detached from institutions, networked whith friends [información en página web]. Recuperado de http://www. pewsocialtrends.org/2014/03/07/millennials-in-adulthood/

Comando de Personal [COPER]. (2015). Estadísticas de retiro voluntario de personal militar.Archivo Comando de Personal del Ejército Nacional.

Correa, A., Reyes, J. \& Reyes, M. (2019). Ejército Nacional frente a la retención del talento humano millennial (Tesis de Especialización). Universidad Piloto de Colombia, Bogotá, D. C., Colombia.

Giménez, R. (2007). Aplicación de la teoría fundamentada (grounded theory) al estudio del proceso de creación de empresas. En Decisiones basadas en el conocimiento y en el papel social de la empresa (XX Congreso anual de Aedem) (vol. 2. Comunicaciones). Recuperado de https://dialnet.unirioja.es/servlet/articulo? codigo=2499458

Gracia, F., Peiró, J. M. \& Mañas, M. A. (2007). El contrato psicológico en la administración pública: derechos y obligaciones del empleado público desde su propia perspectiva. Revista de Psicología del Trabajo y de las Organizaciones, 23 (3), 389-418. Recuperado de https://journals.copmadrid.org/jwop/art/8065d07da4a77621450aa84fee5656d9

Núnez, J. M. (2013). La conciliación entre la vida personal y la profesional. Empresa y Humanismo, 10 (1), 181-208.

Organismo Internacional de Juventud [OIJ]. (2016). Millenials ¿ Una categoría útil para identificar a las juventudes iberoamericanas? [documento en internet]. Recuperado de 
https://oij.org/wp-content/uploads/2017/08/Sobre-la-categori\%CC\%81a-MillennialsVersi\%C3\%B3n-web.pdf

Robbins, S. \& Judge, S. P. (2009). Comportamiento organizacional. México: Pearsons Educación.

Sampieri, R., Fernández, C. \& Baptista, P. (2018). Metodología de la investigación. México: McGraw Hill.

Shein, E. H. (1980). Organizational psychology. Englewod Cliffs: Pretence-Hall.

Tena, G. T. (2002). El contrato psicológico: relación laboral empresa-trabajador. Acciones e Investigaciones Sociales, (15), 85-107. Recuperado de https://dialnet.unirioja.es/ descarga/articulo/284117.pdf

Zauza, A. (2010). El proyecto de autorrealización cambio, curación y desarrollo. Espańa: CLB Universitario. 